\title{
Contribution of Body Movements on the Heart Rate Variability During High Intensity Running
}

\author{
Iman Alikhani ${ }^{1}$, Kai Noponen ${ }^{1}$ and Tapio Seppänen ${ }^{1}$
}

\begin{abstract}
We studied the association between the heart rate variability (HRV) and the subject's movement during high intensity running. HRV is affected by movement, and this phenomena is known as cardiolocomotor coupling (CLC). Characterization of movement related components on the HRV spectrogram is a principal step toward meaningful interpretation of autonomic nervous system (ANS) activity. According to the literature, the aliases of the first and second harmonics of the cadence frequency are the main contributors affecting HRV. Instead, we found out that there is another aliasing component containing significant power in the HRV spectrogram. The source of this component might be the arm swings, torso movement or any other mechanical movement along the horizontal axis, orthogonal to the cadence direction. Our results show that in 13 out of 22 subjects the spectral HRV component arising from the alias of the second harmonic of cadence frequency (vertical acceleration) accommodates significantly less energy than the component related to the alias of the first harmonic of horizontal acceleration. Therefore, neglecting this component and/or considering the second harmonic of the cadence frequency as more dominant one is not always a valid assumption.
\end{abstract}

\section{INTRODUCTION}

Heart rate variability (HRV) has been one of the key indicator of cardiac and autonomic nervous system (ANS) functionality and has been studied in different contexts, targeting physiological evaluation or diagnostic of cardiac diseases [1]. Although the functionality of ANS at rest condition can be inferred from HRV power spectral density, such an interpretation is not trivial in the exercise context. There are variety of causes contributing to the complexity of HRV spectrogram during uncontrolled conditions, particularly high intensity running. Non-stationary essence of the exercise, variable mean heart rate (HR), and, importantly, introduction of cardiolocomotor coupling (CLC) related components[2], [3] into the HRV affect the spectral contents throughout the measurements.

Traditionally, the frequency spectrum of HRV is divided into two main constituents, high frequency (HF) and low frequency (LF) components. In rest, the HF component is defined as the energy within the range of $[0.15 \mathrm{~Hz}, 0.4 \mathrm{~Hz}]$, and is considered to originate mainly from the parasympathetic activity of ANS. Correspondingly, the LF component is defined as the energy within the $[0.04 \mathrm{~Hz}, 0.15 \mathrm{~Hz}]$ frequency band that is mediated by the sympathetic and parasympathetic activity of ANS [1]. Having said that, the spectral energy distribution of HRV during high intensity

\footnotetext{
${ }^{1}$ Physiological Signal Analysis Team, Center for Machine Vision and Signal Analysis, University of Oulu, Oulu, Finland \{iman.alikhani, kai.noponen, tapio.seppanen\}@oulu.fi
}

running is highly influenced by other factors arising from the aforementioned causes leading to the redefinition of the spectral interpretation. The breathing frequency doubles or even triples during high intensity exercise, and the component induced by respiration takes place at frequencies above the traditional HF bandwidth of HRV [3].

The characterization and localization of HRV power spectral density over time enhances our understanding about the ANS and cardiac functionality in exercise contexts, which is strongly appealing for sport physicians and athletes. Recently, a few studies have been conducted towards understanding the influence of stride and pedaling frequency on the HRV spectrogram during running and cycling [2], [4], [5]. The principal association amongst these papers is the significant power of CLC components over HRV spectrogram. They reported that the power associated to the CLC components in the HF spectrum of HRV is not quantitatively negligible. For instance, the median of $22 \pm 7 \%$ of total HRV power in [2] during running, and also $10 \%-30 \%$ during cycling and running in [4] are reported. In addition, in some cases, the power of CLC-related components might surpass that of the respiratory-related ones, which can mislead the interpretation of spectral analysis and cardiac functionality.

In this study, we approach the characterization and localization of disruptive movement artifact on the HRV spectrogram from a different perspective. We show that unlike the reported studies, cadence frequency and its harmonics are not the only main contributors to the HRV signal, but running style and other dominant rhythms of the body exert influence on the HRV spectrogram. What is more, we expect that in some cases they might contain many times more power than the cadence harmonics.

\section{Method}

\section{A. Database}

In total, 22 subjects participated in an incremental running protocol on a treadmill. The treadmill started at $2.7 \mathrm{kmh}^{-1}$ and is incremented every three minutes to $4,5.5,6.8,8$, 8.8 and $9.6 \mathrm{kmh}^{-1}$ till voluntary exhaustion of subjects. A three-channel accelerometer plus a single-channel ECG electrode monitored the movement and cardiac activity. ECG signal was up-sampled to $1 \mathrm{kHz}$. At the same time the respiratory frequency was measured using a spirometer with $1 \mathrm{~Hz}$ sampling frequency. We have used the last 100second portion of the signal, which is the highest intensity of the exercise and close to subject's maximal HR. The physiological background of subjects are provided in Table I. 


\section{B. Signal Preprocessing}

To characterize the CLC components within the spectrogram of HRV, the signal of a three-channel accelerometer attached to the subject's chest is processed. The hypothesis is that the components contaminating the HRV spectrogram during exercise, originate from the mechanical movement of subject based on his/her individual running style. Thus, the CLC components in the HRV spectrogram are not entirely characterized by the stride frequency alone. The three acceleration channels carry not only the cadence frequency dominating the vertical channel, but also the movement of his/her chest along the horizontal (frontal) and sagittal axes.

The acceleration signals are smoothed and their baseline is reduced. Their frequency content are mostly a single dominant frequency band and its harmonics. It means that most of the energy is accommodated in a very narrow band. Therefore, the central frequency of this narrow band is handpicked from the three channels of accelerometer spectrograms, which is formed by short time Fourier transform (STFT) model, down-sampled to eight $\mathrm{Hz}$ and expressed as $c_{i}^{j}(n), i=\{x, y, z\}, j=\{1,2, \ldots\}$. Subscript $i$ and superscript $j$ represent the channel and the harmonic number of the band, respectively. Figure 1 illustrates a 200-second sample accelerator spectrograms.

R-waves are extracted from the single-channel ECG signal. Beat occurrence time series is taken for the construction of instantaneous HR signal $(h r(k))$. Outliers and ectopic beats are identified using the method explained in [6], and interpolated using spline interpolation of neighboring controlled beats. Then, $h r(k)$ is sampled at eight Hertz using linear interpolation and is indicated as $h r(n)$. The baseline of $h r(n)$ is computed using a second-order Savitzky-Golay filter with

TABLE I

THE PHYSIOLOGICAL META DATA OF SUBJECTS PARTICIPATED IN THIS EXPERIMENT IS GIVEN IN THIS TABLE. BMI AND FS STANDS FOR BODY MASS INDEX AND THE FINAL SPEED OF THE SUBJECTS, RESPECTIVELY

\begin{tabular}{|c|c|c|c|c|}
\hline ID & Gender & Age $(\mathrm{Y})$ & $\mathrm{BMI}\left(\mathrm{kg} \mathrm{m}^{-2}\right)$ & $\mathrm{FS}\left(\mathrm{kmh}^{-1}\right)$ \\
\hline 1 & M & 37 & 20.7 & 8.8 \\
\hline 2 & F & 28 & 21.7 & 8 \\
\hline 3 & M & 31 & 25.5 & 8 \\
\hline 4 & F & 24 & 20.6 & 8 \\
\hline 5 & F & 25 & 17.9 & 8.8 \\
\hline 6 & M & 33 & 23.7 & 8.8 \\
\hline 7 & F & 32 & 23.2 & 6.8 \\
\hline 8 & F & 45 & 18.5 & 8 \\
\hline 9 & M & 29 & 23.7 & 8.8 \\
\hline 10 & M & 30 & 31.0 & 6.8 \\
\hline 11 & F & 26 & 25.9 & 6.8 \\
\hline 12 & M & 46 & 28.9 & 6.8 \\
\hline 13 & M & 23 & 32.5 & 6.8 \\
\hline 14 & M & 38 & 26.2 & 5.5 \\
\hline 15 & F & 39 & 32.8 & 5.5 \\
\hline 16 & M & 29 & 19.6 & 9.6 \\
\hline 17 & M & 29 & 28.0 & 6.8 \\
\hline 18 & F & 37 & 27.9 & 6.8 \\
\hline 19 & M & 28 & 27.9 & 8 \\
\hline 20 & M & 51 & 19.6 & 8 \\
\hline 21 & M & 41 & 34.9 & 6.8 \\
\hline 22 & M & 39 & 19.0 & 8 \\
\hline
\end{tabular}

a 200-sample-window. This baseline is essentially the local mean HR, denoted as $h r_{m}(n)$. The trend of instantaneous HR is removed by the baseline, and HRV signal is constructed as $h r v(n)=h r(n)-h r_{m}(n)$. The normalization proposed in [7] is then applied to attenuate the influence of varying mean HR on the HRV signal by $p(n)=\frac{h r v(n)}{h r_{m}(n)}$. Eventually, $p(n)$ is considered for further spectral analysis.

\section{Spectral Characterization}

The preprocessed signal, $p(n)$, is analyzed by the smoothed pseudo Wigner-Ville distribution (SPWVD) timefrequency analysis with 1024 frequency bins. Elliptical exponential parameters of SPWVD are chosen as $V_{0}=0.03$, $\tau_{0}=0.09$ and $\lambda=0.3$ [8]. SPWVD gives a high resolution in time and frequency and suits our spectral processing.

According to [2], during exercise, the intrinsic sampling frequency of HRV signal is essentially handled by the local HR at each time instant. It means that the meaningful physiological components can be situated within the frequency range of $\left[0 \mathrm{~Hz}, h r_{m}(n) / 2 \mathrm{~Hz}\right]$. Thus, during exercise, the HF bandwidth of HRV signal is redefined as $\left[0.15 \mathrm{~Hz}, h r_{m}(n) / 2 \mathrm{~Hz}\right]$, rather than classical HF bandwidth of $[0.15 \mathrm{~Hz}, 0.40 \mathrm{~Hz}]$.

The frequency components of the $x, y$ and $z$ channels of the acceleration, illustrated in Figure 1, contaminate the HRV spectrogram. The bright narrow frequency band in the top sub-figure of Figure $1\left(c_{x}^{1}\right)$, accommodates the cadence frequency component, which contains highest power in the accelerometer signal. It is the most dominant energy band in the $x$-axis. Although its second harmonic $\left(c_{x}^{2}\right)$ contains notable amount of energy, $y$-channel spectrogram (middle sub-figure) also offers a considerable energetic band $\left(c_{y}^{1}\right)$ in the latter portion of the exercise. Thus, there is another component apart from the cadence and its harmonics, reflecting on the HRV spectrogram and contains significant power compared to the second harmonic of the cadence frequency. Hence, it may have major contribution to the HRV spectrogram.

Supposing linearity, the components below the Nyquist frequency of HRV signal $\left(h r_{m}(n) / 2\right)$ basically map to the same frequencies in the HRV spectrogram but with some coupling strength that depends on instantaneous conditions of the subject and measurement. Moreover, the components over the Nyquist frequency of HRV signal fold back to a lower frequency as the result of aliasing in the HRV spectrogram due to sampling happening at the HR. As noted, it is expected that the actual strength of transfer between accelerometer components and the HRV spectrogram can vary both in time and frequency. In any case, the aliasing phenomenon disturbs the interpretation of respiratory-origin components and $\mathrm{HF}$ variation of HRV signal. In some cases, depending on the subject's cadence style and the severity of impact inserted through the body, CLC-related components and its aliases could be more energetic than the RSA components.

In a linear system with aliasing, the component frequencies represented as $c_{i}^{j}(n), i=\{x, y, z\}, j=\{1,2, \ldots\}$, map to the HRV spectrogram frequencies with the function of 

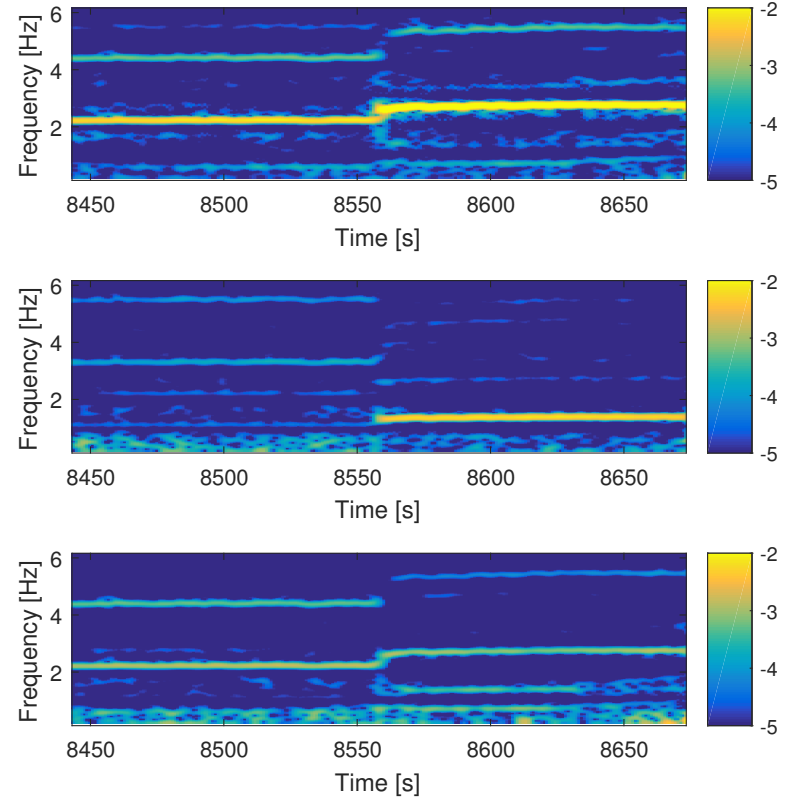

Fig. 1. A sample accelerator spectrogram. Respectively, from top to bottom sub-figures, channels $x, y$ and $z$ are depicted. All the spectrograms are shown on the same logarithmic scale. There is a sudden change near the time stamp 8550 s, corresponding to a velocity increment. The top sub-figure shows the cadence frequency over the yellow narrow band and its second harmonic (This channel generate relatively more power compared to other channels). The second spectrogram, particularly the later halftime (last 100 seconds), illustrates another powerful narrow band situated in different frequency The bottom sub-figure ( $z$-channel), is a superposition of $x$ and $y$ channel spectrograms.

Equation 11 disregarding the coupling strength. Figure 2 illustrates this folding frequency as a function of $c_{i}^{j}(n)$. It basically shows in which frequency of HRV spectrogram, the accelerometer frequencies are related to each other. The mapping frequency at each time instant depends on the $c_{i}^{j}, i=\{x, y, z\}, j=\{1,2, \ldots\}$ and subject's HR at that time instant. The mapping formula can be found with the Equation 1

$$
A_{i}^{j}(n)=\left|c_{i}^{j}(n)-\left[\frac{c_{i}^{j}(n)}{h r_{m}(n)}\right] \times h r_{m}(n)\right|,
$$

where $i=\{x, y, z\}, j=\{1,2, \ldots\}$, and $[x]$ means the nearest integer to $x$. All the units are in Hz. $A_{i}^{j}(n)$ corresponds to the mapped signal over the HRV spectrogram.

The corresponding bandwidth of the folded frequencies is $0.125 \mathrm{~Hz}$, according to the equation given in [2], $\Delta F=$ $4\left(\frac{4 F_{s}}{2 K-1}\right)$, where $F_{s}$ is the sampling frequency of the signal and $2 K-1$ is the Hamming window length in SPWVD time frequency-model.

\section{RESULTS}

\section{A. Spectral Analysis}

It is clear that the cadence frequency $\left(c_{x}^{1}(n)\right)$ is a major component folding back to the HRV spectrum. However, it is not the only component related to the subject's movement

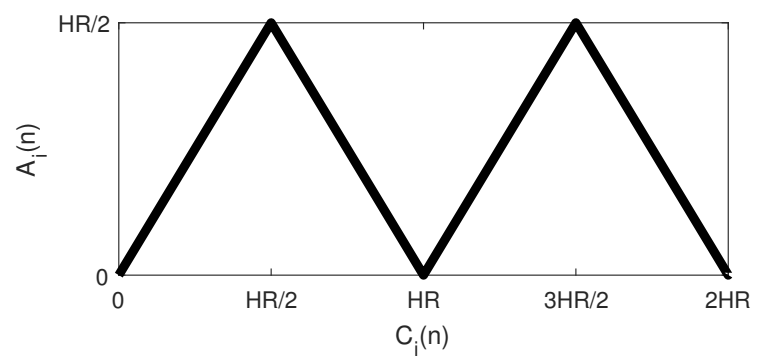

Fig. 2. The mapping function of accelerator frequency to the HRV spectrogram is depicted in this figure. $c_{i}^{j}(n), i=\{x, y, z\}, j=\{1,2, \ldots\}$ at each time instant $(n)$ is given to this function and the corresponding mapped frequency is computed.
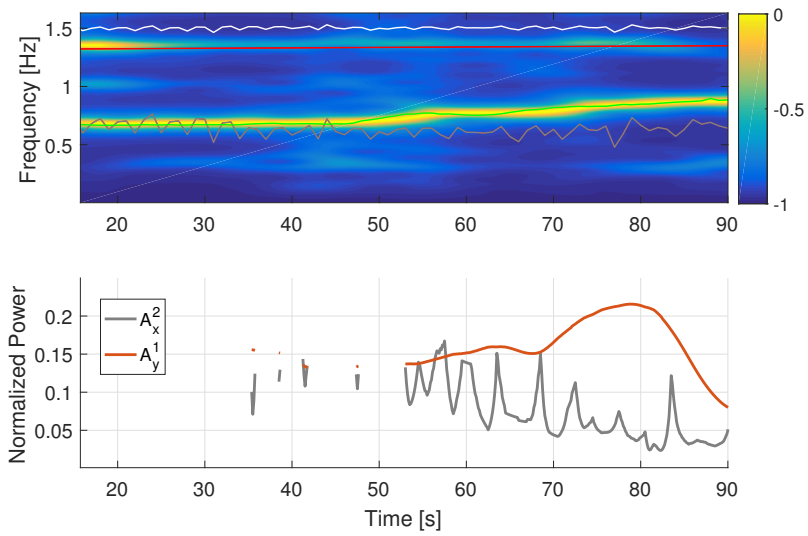

Fig. 3. A normalized spectrogram of a sample $p(n)$ signal (top sub-figure). White solid line is the intrinsic sampling frequency of $\operatorname{HRV}\left(h r_{m}(n) / 2\right)$, green solid line shows the breathing frequency, red and gray solid lines represent the mapped components $A_{y}^{1}$ and $A_{x}^{2}$, respectively. Normalized power over the red and gray bands on the top sub-figure is depicted in the bottom sub-figure. The time instants that the mapped frequency is overlapped with the respiratory frequency is skipped, since the characterization of CLC component in those time instants is not possible.

in the HRV spectrum. In our experiment, we compare the normalized energy, accommodated over the bandwidth of $A_{x}^{2}(n)$ and $A_{y}^{1}(n)$, in order to measure the energy significance over the second harmonic of stride frequency $\left(c_{x}^{2}(n)\right)$ and the first harmonic of $y$-channel $\left(c_{y}^{1}(n)\right)$. We neglect the portion of data, in which the respiratory frequency overlaps with $A_{x}^{2}(n)$ and $A_{y}^{1}(n)$, since the source of energy can not be distinguished easily. Figure 3 shows a sample case with the associated normalized power of the mapped signals. This sample case illustrates that, in most of the time the power of $A_{y}^{1}(n)$ is more than the power linked to $A_{x}^{2}(n)$. In Table II the acquired figures, as well as some general background of the participants is provided for each sample case.

To see if there is a statistically significant difference in the strength of these other two components, a statistical test was performed. Since normality was not guaranteed, we used the non-parametric Wilcoxon signed rank test [9], [10] to confirm whether the medians of normalized powers between the observation pairs were the same. Two sided version of the test with $1 \%$ of significance level was selected. The $p$ values are provided in Table II In all but one of the cases, 
the median normalized powers were statistically different.

\section{TABLE II}

TABLE OF RESULTS, ACQUIRED FROM THE POWER SPECTRAL DENSITY OF HRV SPECTROGRAMS. MEDIAN NORMALIZED POWER OF EACH

SAMPLE IS GIVEN IN THE TABLE. THE GRAY ROWS SHOWS THE SAMPLES, IN WHICH THE $A_{y}^{1}(n)$ BAND ACCOMMODATES MORE ENERGY THAN $A_{x}^{2}(n)$.

\begin{tabular}{|c|c|c|c|}
\hline ID & $P_{A_{x}^{2}}(\mathrm{n} . \mathrm{u})$ & $P_{A_{y}^{1}}(\mathrm{n} . \mathrm{u})$ & $p$-value \\
\hline 1 & 0.046 & 0.025 & 0.000 \\
\hline 2 & 0.096 & 0.120 & 0.000 \\
\hline 3 & 0.063 & 0.156 & 0.000 \\
\hline 4 & 0.120 & 0.105 & 0.000 \\
\hline 5 & 0.098 & 0.112 & 0.000 \\
\hline 6 & 0.089 & 0.110 & 0.000 \\
\hline 7 & 0.107 & 0.136 & 0.000 \\
\hline 8 & 0.148 & 0.039 & 0.000 \\
\hline 9 & 0.058 & 0.039 & 0.000 \\
\hline 10 & 0.075 & 0.108 & 0.000 \\
\hline 11 & 0.042 & 0.116 & 0.000 \\
\hline 12 & 0.057 & 0.152 & 0.000 \\
\hline 13 & 0.084 & 0.204 & 0.000 \\
\hline 14 & 0.086 & 0.094 & 0.000 \\
\hline 15 & 0.085 & 0.058 & 0.002 \\
\hline 16 & 0.040 & 0.119 & 0.000 \\
\hline 17 & 0.064 & 0.104 & 0.000 \\
\hline 18 & 0.069 & 0.063 & 0.016 \\
\hline 19 & 0.093 & 0.070 & 0.000 \\
\hline 20 & 0.239 & 0.014 & 0.000 \\
\hline 21 & 0.068 & 0.028 & 0.000 \\
\hline 22 & 0.067 & 0.068 & 0.000 \\
\hline
\end{tabular}

As it is highlighted in Table II] The figures show that, considering the cadence frequency and its harmonics are not sufficient for characterization of CLC components over the HRV spectrogram. Despite the association of our findings in some subjects of the experiment (e.g. ID 8, 20 and 21) with the results reported in [4], [2], in the sense that the harmonics of cadence are the major contributors of CLC effect into the HRV spectrogram, there are other subjects (e.g. ID 3, 11, 12 and 13) with times more power in the $A_{y}^{1}(n)$ frequency. This finding reveals that in order to distinguish and attenuate the effect of CLC in the HRV during high intensity running, the entire rhythm and movement of subjects should be modeled.

\section{CONCLUSION}

High intensity running introduces cardiolocomotor coupling components to the HRV signal. These components distract the understanding of ANS activities. Thus, the identification of these elements in a HRV spectrogram is a preliminary step before any analysis regarding the HRV features. Although, the cadence of the subject is a major cause of CLC components, there are other constituents with different frequencies reflected in the HRV spectrogram. Especially there often is an aliasing component related to the dominant movement along the horizontal axis (i.e. torso movement or arm swing) that in some cases (13 out of 22 subjects) contains more energy than the alias of the second harmonic of cadence. This finding of ours contradicts or at least complement some earlier work, namely [2], [4]. However, depending on the intensity of exercise and running style of athletes, different components from the movements could be mapped over the HRV spectrogram. Thus, it underlines the need for the mechanical modeling of subject's movements during running for comprehensive characterization of disturbances in the HRV signal. Finally, it is worth noting that HRV behavior should be interpreted cautiously throughout the exercise context specifically running, since the error margins are larger due to the introduced disruptive components.

\section{REFERENCES}

[1] T. F. of the European Society of Cardiology et al., "Heart rate variability standards of measurement, physiological interpretation, and clinical use," Eur Heart J, vol. 17, pp. 354-381, 1996.

[2] R. Bailón, N. Garatachea, I. de la Iglesia, J. A. Casajús, and P. Laguna, "Influence of running stride frequency in heart rate variability analysis during treadmill exercise testing," IEEE transactions on biomedical engineering, vol. 60, no. 7, pp. 1796-1805, 2013.

[3] A. E. Aubert, B. Seps, and F. Beckers, "Heart rate variability in athletes," Sports medicine, vol. 33, no. 12, pp. 889-919, 2003.

[4] A. Hernando, D. Hernando, N. Garatachea, J. Casajús, and R. Bailón, "Attenuation of the influence of cardiolocomotor coupling in heart rate variability interpretation during exercise test," in 2015 37th Annual International Conference of the IEEE Engineering in Medicine and Biology Society (EMBC). IEEE, 2015, pp. 1508-1511.

[5] G. Blain, O. Meste, A. Blain, and S. Bermon, "Time-frequency analysis of heart rate variability reveals cardiolocomotor coupling during dynamic cycling exercise in humans," American Journal of Physiology-Heart and Circulatory Physiology, vol. 296, no. 5, pp. H1651-H1659, 2009.

[6] I. Alikhani, K. Noponen, A. Hautala, R. Ammann, and T. Seppnen, "Spectral data fusion for robust ecg-derived respiration with experiments in different physical activity levels," in Proceedings of the 10th International Joint Conference on Biomedical Engineering Systems and Technologies - Volume 5: HEALTHINF, (BIOSTEC 2017), INSTICC. SciTePress, 2017, pp. 88-95.

[7] R. Bailón, G. Laouini, C. Grao, M. Orini, P. Laguna, and O. Meste, "The integral pulse frequency modulation model with time-varying threshold: application to heart rate variability analysis during exercise stress testing," IEEE transactions on biomedical engineering, vol. 58, no. 3, pp. 642-652, 2011.

[8] M. Orini, R. Bailón, L. T. Mainardi, P. Laguna, and P. Flandrin, "Characterization of dynamic interactions between cardiovascular signals by time-frequency coherence," IEEE transactions on biomedical engineering, vol. 59, no. 3, pp. 663-673, 2012.

[9] M. Hollander, D. A. Wolfe, and E. Chicken, Nonparametric statistical methods. John Wiley \& Sons, 2013.

[10] J. D. Gibbons and S. Chakraborti, Nonparametric statistical inference. Springer, 2011. 